\title{
Qualidade de Vida Familiar na Deficiência Intelectual: Revisão Sistemática de Estudos
}

\author{
Raquel Alveirinho Correia ${ }^{1,2, *} \&$ Maria João Seabra-Santos ${ }^{1}$ \\ ${ }^{1}$ Faculdade de Psicologia e Ciências da Educação da Universidade de Coimbra, Coimbra, Portugal \\ ${ }^{2}$ Associação Portuguesa de Pais e Amigos do Cidadão Deficiente Mental de Castelo Branco, Castelo Branco, Portugal
}

\begin{abstract}
RESUMO - A qualidade de vida familiar (QdVF) em famílias de pessoas com deficiência intelectual (DI) tem interessado investigadores, sugerindo potencialidades em termos de avaliação e intervenção. O presente artigo realizou uma revisão sistemática dos estudos empíricos que analisam a QdVF em famílias de pessoas com DI. Efetuou-se uma pesquisa bibliográfica num portal de bases de dados e recorreu-se a critérios de inclusão previamente definidos. Do total de 32 estudos selecionados, 15 recorreram ao instrumento FQOLS-2006, focando-se na análise das dimensões e domínios da mesma; 11 ao Beach Center FQOL Survey, relacionando o constructo com diversas variáveis; e seis a outras formas de avaliação, essencialmente qualitativas. Assim, a QdVF mostra-se pertinente para compreender e intervir com famílias de pessoas com DI.
\end{abstract}

PALAVRAS-CHAVE: qualidade de vida familiar, deficiência intelectual, escalas de avaliação

\section{Family Quality of Life in Intellectual Disability: A Systematic Review of Studies}

\begin{abstract}
Family quality of life (FQoL) for families with members who have an intellectual disability (ID) has recently captured the interest of researchers and demonstrated potential for evaluation and intervention. This paper intends to revise systematically empirical studies that analyze FQoL in families with members having ID. A bibliographic search was carried out in a database and previously defined inclusion criteria were used. From a total of 32 studies, 15 used the FQOLS-2006 that focused on the analysis of their dimensions and domains, 11 the Beach Center FQOL Survey that related the construct to other individual and family variables and six other methods to evaluate FQoL. Thus, FQoL is relevant to understand and intervene with families of persons with ID.

KEYWORDS: Family quality of life, intellectual disability, measurement instruments
\end{abstract}

De acordo com a American Association of Intellectual and Developmental Disabilities (AAIDD), a deficiência intelectual (DI) é caracterizada por limitações significativas no funcionamento intelectual e no comportamento adaptativo, que se manifestam nas competências adaptativas em termos conceituais, sociais e práticos (AAIDD, 2011). Alguns autores (e.g., Baum, 2006; Hatton, 2012) consideram que esse é um conceito construído socialmente e sujeito a algumas variações na sua definição e identificação, o que justifica a pertinência de se adotar uma abordagem multidimensional no seu estudo que considere o contexto no qual a pessoa com DI se insere (AAIDD, 2011). O termo contexto deve aqui ser entendido à luz da perspetiva ecológica (Bronfenbrenner,1977), que considera a pessoa em relação com os seus contextos de vida (família, trabalho, escola, instituições) e reconhece que esses são influenciados por contextos mais amplos, como a cultura e a etnia (Fredman, 2006).

A par dessa revisão do conceito de deficiência intelectual, o conceito de qualidade de vida também tem ganhado mais destaque e amplitude na investigação e intervenção nessa área (Schalock \& Verdugo, 2003; Schalock et al., 2002). Para Schalock e Verdugo (2003) e Schalock et al. (2002), mais importante do que definir a QdV individual, é identificar as suas dimensões e indicadores, considerando-os como um conjunto de fatores que devem potenciar o bem-estar

*E-mail: raquel.a.correia@gmail.com 
pessoal, mantendo o indivíduo integrado no seu contexto cultural e étnico, de forma a aumentar o grau de controle pessoal e de oportunidades individuais. Assim, tendo em conta a pertinência desse conceito, os serviços que prestam apoio na área devem pautar as suas práticas pela melhoria da QdV de pessoas com DI (Keith, 2007), sendo que a avaliação da qualidade da intervenção dos serviços deve ter em conta a QdV percecionada pelas pessoas com DI com quem intervêm (Tamarit, 2006).

Em continuidade, Verdugo, Schalock, Keith e Stancliffe (2005) mencionaram que quando se pretende avaliar e estudar a QdV a nível individual, deve-se ter em conta as seguintes diretrizes: trata-se de um conceito multidimensional, que envolve componentes objetivas e subjetivas; a recolha dos dados deve ocorrer diretamente com a pessoa com DI e/ou a sua família; na investigação deve ser usado um pluralismo metodológico que envolva quer medidas objetivas, quer subjetivas, recorrendo a designs de investigação multivariados para avaliar a forma como as características pessoais e as variáveis ambientais se relacionam com a QdV da pessoa avaliada; ao planificar investigações e implementar avaliações da QdV, deve-se procurar aumentar o envolvimento da própria pessoa com DI; é útil incorporar uma perspetiva sistêmica que capte os múltiplos ambientes que têm impacto a nível micro, meso e macro e ser sensível às diferenças culturais.

Esses diversos aspectos remetem à importância de considerar a qualidade de vida não só no plano individual, mas também no plano familiar. Contudo, se a QdV individual está amplamente estudada na área da DI (Schalock \& Verdugo, 2003), o mesmo não acontece com a QdVF, que só recentemente começou a ser investigada nessa área. Assim, o presente artigo tem como objetivo rever os resultados de estudos empíricos acerca da QdVF em famílias de pessoas com DI, a fim de criar um ponto de partida para futuras investigações.

\section{FAMÍLIAS DE PESSOAS COM DEFICIÊNCIA INTELECTUAL}

Crianças com deficiência podem nascer em qualquer tipo de família e sendo esse um acontecimento muitas vezes não antecipado, a maioria dessas famílias aprende a aceitá-lo, manifestando-se, no entanto, frequentemente pouco preparada e sem conhecimentos especializados para enfrentar os desafios "especiais" colocados por uma criança com incapacidade (Seligman \& Darling, 2007). Uma família com pessoas com deficiência é, antes de tudo, uma família que procura ter acesso e usufruir dos benefícios da sua comunidade, residir em uma casa e bairro com segurança, viver, crescer e experienciar todas as alegrias e tristezas comuns em qualquer família, de forma a permanecer emocional e fisicamente saudável (Zuna, Summers, Turnbull, Hu \& Xu, 2010). Salienta-se que, ao longo do seu ciclo de vida, as famílias de pessoas com DI apresentam as mesmas tarefas desenvolvimentais que as restantes famílias, às quais acrescem outras tarefas específicas relacionadas com a DI, o que acrescenta riscos e desafios particulares com que essas famílias se deparam (Carr \& O'Reilly, 2007). Desse modo, tal como ocorre com todas as famílias, as famílias de pessoas com DI também podem ser muito diferentes umas das outras (Leal, 2008).

Com o objetivo de proporcionar uma melhor $\mathrm{QdV}$, verifica-se que cada vez mais crianças e adultos com DI recebem cuidados em ambiente familiar. Essa prática permite que as pessoas com DI levem uma vida mais próxima do que é normativo, possibilitando o envolvimento com os membros da sua família e que esses se comprometam com os cuidados a serem prestados. Nesse sentido, os profissionais podem melhorar os seus serviços adotando atitudes e práticas centradas na família (Leal, 2008).

De fato, desde sempre as famílias representaram os pilares das pessoas com DI, constituindo-se como os principais prestadores de cuidados e apoios. Somente nos dois últimos séculos surgiram opções de prestação de cuidados exclusivamente fora da família, levando, muitas vezes, à institucionalização da pessoa com DI. No entanto, nos últimos anos, devido a mudanças que levaram à defesa de políticas e práticas que procuram promover o viver em comunidade e a valorização dos cuidados prestados pelas famílias, essas voltaram a assumir um papel mais central no apoio a pessoas com DI (Brown \& Brown, 2004a).

Também, na Convenção das Nações Unidas sobre os direitos da Pessoa com Deficiência (Instituto Nacional para a Reabilitação, 2010), a família assume um papel de destaque. Nessa Convenção, salienta-se o papel essencial das famílias e que essas têm "direito à proteção pela sociedade e pelo Estado e que as pessoas com deficiência e os membros da sua família devem receber a proteção e assistência necessárias para permitir às famílias contribuírem para o pleno e igual gozo dos direitos das pessoas com deficiência” (p. 9).

\section{QUALIDADE DE VIDA FAMILIAR}

Tendo em conta que a família é uma rede de relações recíprocas com as suas próprias características e necessidades e, não obstante, se reconhecer a individualidade dos seus elementos, importa olhar para a unidade familiar com a sua própria história compartilhada, valores, tradições, objetivos, crenças e prioridades (Leal, 2008). Nesse contexto, faz sentido olhar a QdV não só a um nível individual, mas também familiar. Zuna et al. (2010) definem a Qualidade 
de Vida Familiar (QdVF), em famílias de pessoas com DI, como uma perceção subjetiva de bem-estar da família, para a qual contribuem todos os seus membros e em que as necessidades individuais e familiares interagem. Por sua vez, Park et al. (2003) referem que a QdVF é assegurada quando as necessidades da família são acauteladas e os seus elementos apreciam a sua vida em conjunto, como uma família e como tendo a oportunidade de realizar atividades que consideram importantes.

Brown e Brown (2004b) definiram como aspectos caracterizadores da QdVF: a sua natureza dinâmica e holística, pois todos os aspetos da vida e todos os que influenciam a QdVF estão ligados e influenciam-se mutuamente; o seu caráter desenvolvimental, o que implica olhar para as famílias e indivíduos através do tempo, reconhecendo que cada período desenvolvimental influencia o desenvolvimento futuro; o fato de assentar na perceção e nos valores dos membros da família, de apresentar grande variabilidade entre as famílias, de influenciar a autoimagem da família e de refletir-se na relação com os contextos em que a família se inclui. A QdVF é, assim, multifacetada, à medida em que pode ser melhor pensada como um termo amplo e abrangente, dentro do qual numerosos outros conceitos estão contidos (Brown \& Brown, 2004b). Segundo Samuel, Rillota e Brown (2012), as características anteriormente definidas por Verdugo et al. (2005) para a QdV individual da pessoa com DI e descritas anteriormente, parecem também ser altamente relevantes para medir a QdVF.

$\mathrm{Na}$ ausência de outras teorias acerca da QdVF, Zuna et al. (2010) propuseram um modelo unificador que procurou representar as relações entre múltiplas variáveis que têm impacto na QdVF de famílias com pessoas com DI (e.g., características demográficas dos seus membros, características e dinâmicas familiares, serviços e apoios de que beneficiam ou os sistemas - de saúde, de justiça - políticas e programas) e servir como orientador de investigações futuras. Essa teoria enquadra-se nos princípios sistémicos do Modelo do Desenvolvimento Humano de Bronfenbrenner (1977), pois compreende desde conceitos mais proximais, até outros mais distais e todos esses conceitos se encontram interligados, tornando-o um modelo bastante complexo, tal como são as próprias famílias (Zuna, Turnbull, \& Summers, 2009; Zuna et al., 2010). Para os autores, a QdVF depende das impressões subjetivas dos membros da família acerca da sua satisfação com a qualidade de vida, pelo que não deve ser avaliada por pessoas externas à família, como, por exemplo, prestadores de serviços.

Apesar de a teoria proposta ter sido apoiada em dados de investigação e de revisão de literatura, necessita de pesquisa adicional em algumas das suas componentes, sendo o seu principal objetivo proporcionar aos investigadores um 'mapa de estradas' que guie as suas pesquisas nessa área (uma explicação mais detalhada do modelo de Zuna pode ser consultada em Zuna et al., 2009; Zuna et al., 2010).

Em contextos aplicados à avaliação da QdVF, pode-se assumir um interesse prático, ao permitir conhecer melhor as famílias, as suas áreas prioritárias de intervenção ou quais as áreas fortes que podem ser reforçadas (Summers et al., 2005). Pode, ainda, constituir um indicador apropriado para avaliar serviços e políticas públicas (Schalock, 2004).

\section{PRINCIPAIS INSTRUMENTOS DE AVALIAÇÃO (BEACH CENTER FQOL SURVEY E FQOLS-2006)}

O Beach Center on Disability (Universidade de Kansas) desenvolveu uma escala breve acerca da QdVF na deficiência em famílias americanas de crianças com DI, através de diversas fases de investigação qualitativa (Poston et al., 2003) e quantitativa (Park et al., 2003). A versão preliminar, gerada após uma extensa investigação qualitativa, tinha 10 domínios e 112 itens. A versão final é composta por 25 itens que avaliam cinco domínios da QdVF: Interação familiar, Parentalidade, Bem-estar emocional, Bem-estar físico/ material e Apoio relacionado com a deficiência. É pedido aos respondentes que avaliem todos os itens em relação à Importância que assumem na sua vida familiar e ao grau de Satisfação com a sua qualidade de vida (Summers et al., 2005). A escala foi traduzida para chinês, coreano e espanhol.

O Family Quality of Life Survey (FQOLS-2006) foi desenvolvido originalmente nos EUA por peritos e stakeholders no campo da qualidade de vida de pessoas com deficiência (Brown, Anand, Fung, Isaacs \& Baum, 2003) e procurou, desde o seu início, ter um âmbito internacional e poder ser usado em diferentes etapas do ciclo de vida das famílias. Esse instrumento permitiu identificar nove domínios: Saúde da família, Bem-estar econômico, Relações familiares, Ajuda/apoio de outras pessoas, Apoio prestados pelos serviços a pessoas com deficiência, Influência dos valores, Carreira e preparação da carreira, Lazer e diversão e Interação com a comunidade. As dimensões de avaliação, associadas às questões em cada um dos domínios, incluem: Importância (grau de valorização que a família dá a um determinado elemento), Oportunidades (opções que estão disponíveis para as famílias), Realização (grau no qual as famílias são capazes de realizar e obter o que precisam), Iniciativa (grau no qual as famílias tiram proveito das oportunidades disponíveis), Estabilidade (grau no qual as circunstâncias têm probabilidade de melhorar, piorar ou manter-se) e Satisfação (perceção global acerca de aspetos importantes da vida familiar). O questionário é, assim, constituído por nove secções referentes aos nove domínios, tendo, cada uma das secções, uma parte A (referente a questões descritivas) e uma parte B (em que se pede a classificação relativa às seis dimensões, num total de 63 
itens, numa escala de Likert). Esse instrumento possui, ainda, algumas questões abertas, o que permite a recolha de dados qualitativos (Brown, MacAdam-Crisp, Wang, \& Iarocci, 2006; Isaacs et al., 2007; Werner, Edwards, Baum,
Brown et al., 2009). Atualmente, o FQOLS-2006 é usado em 19 países e foi traduzido para 12 línguas diferentes. Existe uma tradução portuguesa desenvolvida por Benvenuti e Peralta (2012).

\section{METODOLOGIA}

Foi realizada uma pesquisa bibliográfica no portal integrado de bases de dados eletrõnicos em Psicologia e Educação EBSCO Host (PsycINFO / ERIC / PsycARTICLES / Academic Search Premier / Psychology \& Behavioral Sciences Collection / Academic Search Premier / PsycBOOKS /PsycEXTRA/...), usando como palavras-chave "family quality of life" e "disabilit*". Dessa pesquisa inicial, foram extraídos 337 resultados, que foram posteriormente filtrados segundo os critérios ter acesso ao texto integral e fazer parte de uma revista cientifica que possua revisão pelos pares. Acrescentou-se, ainda, o critério de serem estudos empíricos da QdVF na área da deficiência intelectual, excluindo-se todos os que não tivessem como foco essa área ou fossem apenas teóricos. A aplicação desses outros critérios de seleção reduziu a lista inicial de 337 para 21 artigos. As listas de referências bibliográficas dos artigos selecionados foram pesquisadas de acordo com os mesmos critérios. Tendo em conta a sua pertinência para a presente revisão de estudos, foram, ainda, incluídos capítulos do livro Enhancing the Quality of Life of People with Intellectual Disabilities (Kober, 2010), o qual contém textos da revista Social Indicators Research. O processo metodológico encontra-se esquematizado na Figura 1.

No final, foram retidos para análise 32 artigos empíricos, tendo sido divididos nas seguintes categorias, em função dos instrumentos usados para avaliar a QdVF: (1) artigos com recurso ao FQOLS-2006; (2) artigos com recurso à Beach Center FQOL Survey; (3) outros artigos.

Todos os textos incluídos na presente revisão foram resumidos em tabelas, em função do instrumento utilizado e de acordo com: referência e país, design, objetivo, amostra, medidas principais, resultados e limitações. No entanto, dada a extensão dessas tabelas, não serão apresentadas no presente artigo, podendo ser solicitadas diretamente aos autores.

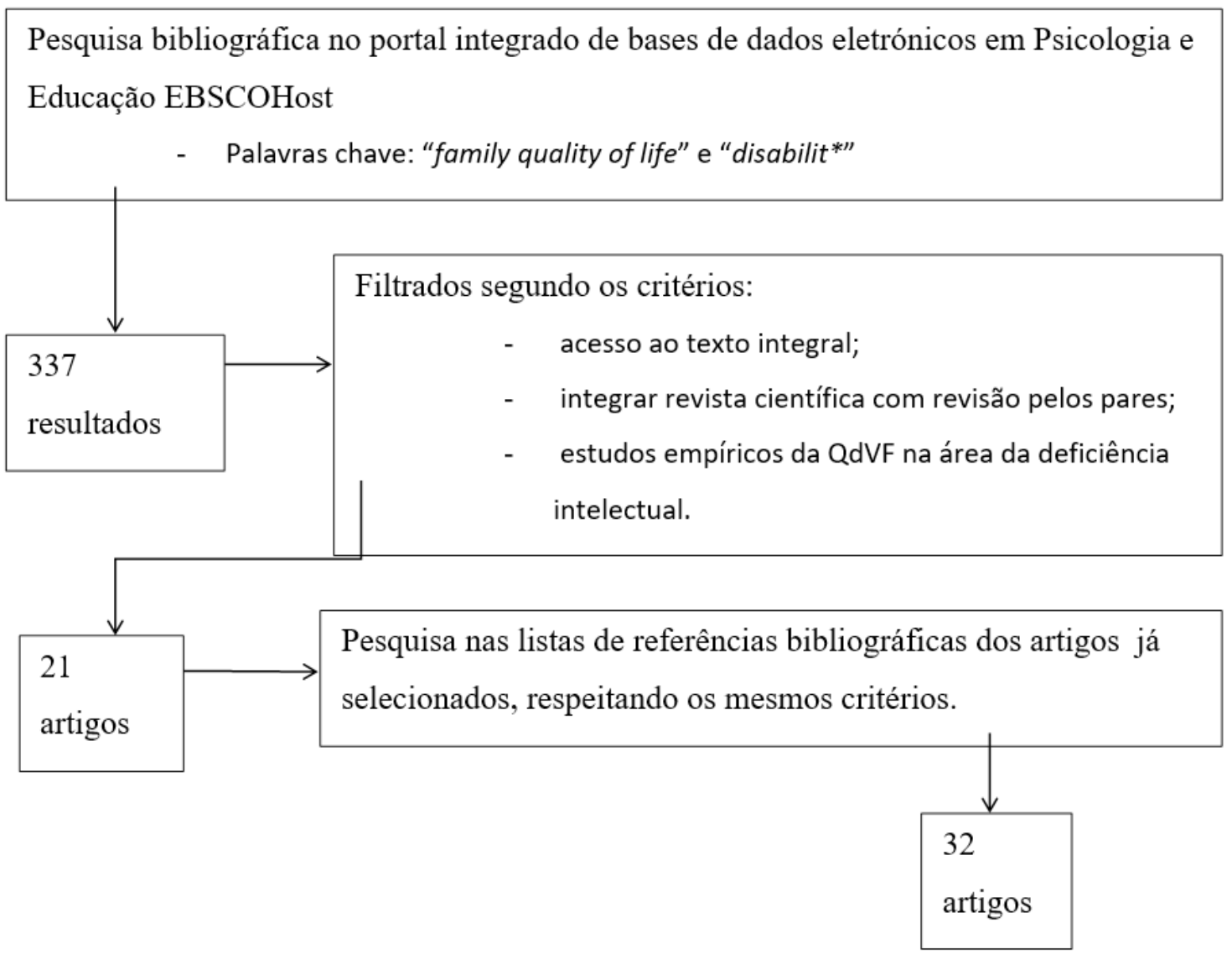

Figura 1. Metodologia utilizada na revisão sistemática acerca de estudos empíricos de qualidade de vida familiar e deficiência intelectual. 


\section{RESULTADOS}

Para a análise dos resultados, os artigos foram divididos em função do instrumento de avaliação sobre o qual incidem. Assim, serão analisados os 15 artigos em que se recorreu ao FQOLS-2006 (e à sua antecessora, FQOLS-2000), seguidamente, os 11 que usaram a Beach Center FQOL Survey e, por fim, os seis estudos empíricos que recorreram a instrumentos diferentes dos anteriores.

\section{Artigos Empíricos com Base no FQOLS-2006}

No que diz respeito aos artigos empíricos em que se recorreu ao FQOLS-2006, três referem-se ao desenvolvimento da escala e à sua evolução, desde a versão preliminar (FQOLS-2000) até à versão atual (Brown et al., 2003; Brown et al., 2006; Werner et al., 2009), sendo registradas as principais alterações efetuadas, que se prenderam com: a reformulação de alguns itens considerados problemáticos e do nome de alguns dos domínios; introdução das dimensões Estabilidade e Importância; divisão das secções da escala em subsecções A e B, em que a primeira apresenta questões descritivas específicas de cada área de vida e a segunda uma questão relativa a cada uma das dimensões avaliadas (Importância, Oportunidades, Realização, Iniciativa, Estabilidade e Satisfação). Foram, ainda, acrescentados itens mais específicos para alguns dos domínios. Ainda de salientar que, no estudo de Brown et al. (2006), com a versão preliminar, a QdVF foi comparada em três tipos de famílias (filho com síndrome de Down, filho com autismo e filho sem deficiência), surgindo diferenças significativas, sendo a QdVF mais elevada nas famílias com filho sem deficiência, quando comparadas com as restantes famílias.

Dez dos estudos apresentados tiveram como objetivo a validação da referida escala nos seguintes países: BósniaHerzegovina (Svraka, Loga \& Brown, 2011), Bélgica (Steel, Poppe, Vandevelde, Van Hove, \& Claes, 2011), Itália (Bertelli, Bianco, Rossi, Scuticchio, \& Brown, 2011), Eslovénia (Čagran, Schmidt, \& Brown, 2011), Israel (Neikrug, Roth, \& Judes, 2011), Malásia (Clark, Brown, \& Karrapaya, 2012), Nigéria (Ajuwon \& Brown, 2012), Austrália (Rillotta, Kirby, Shearer, \& Nettelbeck, 2012), EUA (Samuel, Hobden, LeRoy, \& Lacey, 2012) e Polónia (Ho et al. 2013). Em todos esses estudos, recorreuse ao FQOLS-2006, com algumas especificações, quer na metodologia, quer na análise dos resultados, que se descrevem seguidamente. Todos esses estudos se iniciam (exceto o dos EUA) com uma análise descritiva dos resultados, referindo as médias e desvios-padrão obtidos aos diferentes domínios e dimensões. Em alguns estudos, apresentam-se correlações entre dimensões e entre domínios (Bertelli et al., 2011), entre domínios para cada dimensão (Clark et al., 2012) e apenas entre dimensões (Ajuwon \& Brown, 2012). Existe, também, uma preocupação em complementar os dados quantitativos com informação de natureza qualitativa, quer seja através dos dados recolhidos com o questionário (Čagran et al., 2011; Ajuwon \& Brown, 2012; Rillotta et al., 2012), quer através de uma entrevista (Steel et al., 2011). Apenas um dos estudos anteriormente referidos apresenta os índices de consistência interna (Rillotta et al., 2012), sendo os valores do coeficiente alfa de Cronbach baixos a moderados (Importância $=0,24$; Realização $=0,69$; Oportunidades $=0,79$; Estabilidade $=$ 0,45 ; Satisfação $=0,82$; Iniciativa $=0,48$ ).

O estudo realizado nos EUA (Samuel, Hobden et al., 2012) tem como objetivo analisar os serviços de apoio formais na área da deficiência, em que a análise dos resultados apenas foca no domínio relativo a esse tema do questionário, tendo concluído que as famílias em estudo consideravam os serviços muito importantes para a sua QdVF, mas apenas a metade estava satisfeita com os mesmos, enquanto a outra metade assinalava a necessidade de mais ajuda por parte dos serviços, sendo a falta de informação a maior barreira.

Um estudo australiano procurou avaliar a validade convergente do referido instrumento em relação ao Beach Center FQOL Survey (Rillotta, Kirby, \& Shearer, 2010), tendo concluído que surgem correlações altas entre as áreas que se assemelham nos dois questionários e que os dois instrumentos são igualmente úteis para a avaliação da QdVF, podendo, cada um deles, ser mais ou menos apropriados para fins de investigação ou prestação de serviços, conforme a quantidade de informação necessária, o tempo disponível, os custos de administração e o número de famílias a serem avaliadas. Assim, de acordo com esse estudo, para obter dados mais gerais em menos tempo, deve-se recorrer ao Beach Center FQOL Survey. Caso sejam necessários dados mais detalhados e haja maior disponibilidade de tempo, pode-se recorrer ao FQOLS-2006. Esse estudo salienta, ainda, que se deverá privilegiar a aplicação de qualquer um dos questionários com recurso à entrevista, uma vez que a recolha de informação qualitativa é essencial para compreender o porquê das opiniões expressas pelas famílias. Existe, ainda, um estudo que procura fazer uma análise mais global do instrumento, recorrendo a modelos de equações estruturais, partindo dos dados recolhidos na Austrália, Canadá, Nigéria e EUA (Isaacs et al., 2012). Os dados referentes à Austrália, Canadá e EUA foram trabalhados em conjunto, enquanto os da Nigéria foram trabalhados separadamente, partindo da ideia de que os três primeiros países são semelhantes (em relação ao desenvolvimento econômico, língua e cultura). O ajuste do modelo global sugere que os dados dos três países se ajustam ao modelo de um fator, o que não se verificou com os dados recolhidos na Nigéria. Em relação à precisão, obtiveram-se alfas de Cronbach para os valores globais da QdVF iguais a 0,78 para a amostra da Nigéria e a 0,75 para a dos restantes 3 países. 


\section{Artigos Empíricos com Base no Beach Center FQOL Survey}

Dos artigos que recorreram ao Beach Center FQOL Survey, dois (Poston et al., 2003; Park et al., 2003) referemse ao processo de construção e validação da escala, que se iniciou com um estudo qualitativo ao qual se seguiu um quantitativo para se chegar à versão final do instrumento, que foi ainda aperfeiçoada através de um outro estudo (Hoffman, Marquis, Poston, Summers, \& Turnbull, 2006). Outro trabalho (Wang et al., 2004) procurou relacionar a satisfação e a QdVF com diversas variáveis relativas à família e à própria deficiência, tendo concluído que a severidade da deficiência e o rendimento familiar são preditores significativos da satisfação das mães com a sua QdVF, sendo o primeiro negativo e o segundo positivo. Quanto aos pais, os resultados evidenciaram que a severidade da deficiência é um preditor negativo significativo, no entanto, o rendimento familiar não se mostra significativo, resultado esse que os autores atribuem a limitações na amostra. As diferenças entre pais e mães no que diz respeito à QdVF são também analisadas no artigo de Wang et al.(2006), estudo no qual não foram encontradas diferenças significativas entre esses dois grupos, nem quanto à Importância, nem quanto à Satisfação com a QdVF. Alguns outros estudos pretenderam validar a escala para outros países, nomeadamente Espanha (Verdugo, Córdoba \& Gómez, 2005; Balcells-Balcells, Giné, Guàrdia-Olmos \& Summers, 2011) e China (Hu, Wang \& Fei, 2012), sendo que, no primeiro, obtiveram coeficientes alfa de 0,96 para a Importância e 0,95 para a Satisfação, enquanto no estudo chinês encontraram os valores de 0,94 e 0,91 respetivamente, em ambos os casos revelando uma excelente consistência interna. Ainda no que diz respeito a esse último estudo, os autores comprovaram a estrutura de cinco fatores do constructo da QdVF através de análise fatorial confirmatória e, recorrendo à análise multivariada, constataram que certos aspectos das condições de vida (como habitação e transporte), o rendimento familiar e a severidade da deficiência eram preditores da satisfação com a QdVF. Ainda em um outro estudo, realizado na Austrália (Davis \& Gavidia-Payne, 2009), procurou-se relacionar a QdVF com as características da criança, da família e dos apoios recebidos, sendo que o apoio profissional se mostrou como um dos mais fortes preditores da QdVF, seguindo-se a intensidade percebida dos problemas comportamentais da criança e o apoio da família alargada. Outra variável que se procurou relacionar com a QdVF diz respeito ao local de residência de crianças com deficiência intelectual severa ou profunda, tendo-se concluído que a QdVF surge mais elevada quando as crianças se encontram a viver em casa, do que quando estão em uma instituição, apesar de ambos os grupos avaliarem a sua QdVF como exemplar (McFelea \& Raver, 2012). Um outro estudo procurou conhecer os efeitos de um programa de apoio familiar ao nível da QdVF e do stress parental na Coreia do Sul, tendo concluído que surge uma diferença significativa na QdVF entre o grupo que beneficiou do programa e o que não beneficiou, apesar de não surgirem diferenças significativas quanto ao stress parental (Sung \& Park, 2012).

\section{Artigos Empíricos Não Incluídos nas Categorias Anteriores}

Passamos a apresentar, resumidamente, os resultados dos estudos empíricos que não recorreram a nenhum dos instrumentos anteriormente descritos. Aznar e Castañón (2005) procuraram construir um instrumento de avaliação da QdVF em diversos países da América Latina, composto por 42 indicadores, agrupados em seis domínios, sendo eles: Bem-estar emocional, Desenvolvimento e forças pessoais, Regras de coabitação, Bem-estar físico/material, Vida familiar e Relações interpessoais e comunitárias. Em um estudo qualitativo, com recurso a entrevistas individuais e grupos focais, Jokinen e Brown (2005) procuraram conhecer a perspetiva de pais mais velhos de pessoas com DI, tendo sido identificados os seguintes temas: ser cuidador da família ao longo da vida; reflexões positivas relacionadas com o ser cuidador; descrições da QdVF como normativa ou boa; preocupações com o futuro. Um outro estudo qualitativo (Werner, Edwards, \& Baum, 2009) teve como objetivo estudar o impacto na QdVF da colocação em instituição da pessoa com DI, tendo concluído que as famílias registraram mudanças emocionais positivas após a entrada da pessoa com DI na instituição, apesar de relatos de culpa e preocupação persistentes. Esse estudo apurou, ainda, que a pessoa com DI continua a influenciar a QdVF mesmo depois da sua entrada na instituição, o que mostra que a prestação de cuidados é uma experiência ao longo da vida. Moyson e Roeyers (2012) procuraram conhecer a perspetiva de irmãos de crianças com DI acerca da sua QdVF enquanto irmãos, concluindo que esses conseguem definir a sua própria $\mathrm{QdV}$ e que essa definição difere do conceito de QdVF. Nesse contexto, foram identificados os seguintes domínios de QdV enquanto irmão de criança com DI: atividades em conjunto, compreensão mútua, tempo em privado, aceitação, paciência, bem-estar mútuo, troca de experiências, apoio social e lidar com o mundo exterior. Existe, ainda, um estudo espanhol (Giné, Grácia, Vilaseca \& Balcells, 2010) que descreve uma investigação-ação participativa com recurso a grupos focais e entrevistas para produzir um instrumento para medir a QdVF na Espanha, uma vez que os autores consideraram que traduzir e adaptar a escala americana (Beach Center FQOL Survey) não seria ajustado. Como resultado dessa investigação (Giné et al., 2013), foram criadas duas escalas, uma para famílias cuja pessoa com DI tenha menos de 18 anos e outra se tiver mais de 18. O estudo de validação, que envolveu 1205 famílias espanholas, mostrou que ambas as escalas são consistentes (coeficiente alfa de Cronbach $=0,96 \mathrm{em}$ ambas as escalas) e se apresentam válidas do ponto de vista psicométrico. 


\section{DISCUSSÃO}

A investigação na área da QdVF em famílias de pessoas com DI é bastante recente, tendo sido no ano de 2003 publicados os primeiros artigos nesse âmbito. É de salientar o claro crescimento do interesse dos investigadores nessa temática (Brown, Kyrkou, \& Samuel, 2016), que se traduziu na publicação de diversos artigos e capítulos de livros, incluindo a edição de dois números especiais dedicados à QdVF pela revista Journal of Intellectual Disability Research, no final de 2011 e início de 2012.

Várias explicações podem ser invocadas para compreender esse interesse crescente. Por um lado, uma abordagem com base na QdVF tem o potencial de unir investigação, políticas e práticas (Jokinen, 2006), oferecendo uma visão positiva e compreensiva para avaliar as famílias de pessoas com DI (Samuel, Rillota, \& Brown, 2012). Por outro lado, face à integração de teorias sistêmicas e ecológicas na área da DI, as intervenções individuais têm dado lugar a outras baseadas na família (Leal, 2008), constituindo-se a avaliação da QdVF como uma mais-valia para nortear esse tipo de intervenção. Um outro motivo que justifica o interesse nessa área poderá estar relacionado com o fato de a investigação sobre QdVF ter identificado os domínios que a compõem e as variáveis que a influenciam, que podem ser alvo de uma avaliação e posterior intervenção no sentido de a melhorar.

No que diz respeito à avaliação da QdVF, salienta-se a construção de dois instrumentos: o FQOLS-2006 e o Beach Center FQOL Survey. Com recurso ao FQOLS-2006, encontraram-se estudos em 10 países diferentes, dos cinco continentes. Essa diversidade pode ser explicada atendendo a que os investigadores assumiram a construção do instrumento no contexto de um projeto mais vasto, de âmbito internacional, cujo objetivos consistiam em: desenvolver a conceptualização da QdVF, construir instrumentos e métodos para recolha de informação e aplicar o conhecimento obtido de forma a melhorar a vida das famílias de pessoas com DI (Isaacs et al., 2007).

Quanto ao Beach Center FQOL Survey, destaca-se o rigor metodológico no processo de desenvolvimento do questionário, que se inicia com uma investigação qualitativa exploratória, seguindo-se diversos estudos quantitativos para o seu aperfeiçoamento.

Comparando os estudos que recorreram ao FQOLS-2006 com os que utilizaram o Beach Center FQOL Survey, verificamos que esses últimos procuram relacionar a $Q d V F$ com outras variáveis (ex. rendimento familiar, severidade da deficiência, sexo dos pais, programa de apoio familiar), enquanto os primeiros se limitaram a apresentar os dados fornecidos pelo instrumento. Tal pode dever-se ao fato de o FQOLS-2006 fornecer muito mais informações (relativa a nove domínios e seis dimensões) que o Beach Center FQOL Survey (cinco domínios e duas dimensões). Importa, ainda, referir que ambos os questionários são influenciados pela perspectiva sistémica e ecológica, presente no fato de ambos valorizarem o contexto em que a pessoa e a família se encontram inseridas (Fredman, 2006). Ainda no FQOLS-2006, é possível identificar domínios que correspondem aos três níveis do modelo ecológico e que influenciam a QdV (Schalock \& Verdugo, 2003): microssistema (p. ex., Relações familiares), mesosistema (ex. Interação com a comunidade) e macrosistema (p. ex., Influência dos valores). No que diz respeito às limitações encontradas nos estudos analisados, verificou-se que, na maioria, recorreram a amostras restritas, recolhidas em instituições ou serviços (por conseguinte, excluindo famílias que não recebem qualquer tipo de apoio), o que dificulta a possibilidade de fazer generalizações para a população em geral com DI. Outra limitação prende-se com o fato de a avaliação da QdVF ser realizada apenas por uma pessoa da família (cuidador principal), o que poderá não traduzir as percepções de todos os elementos da família. Outro aspeto que constitui uma limitação da maioria dos estudos analisados (sendo exceções os de Brown et al., 2006, Wang et al., 2004 e Davis \& Gavidia-Payne, 2009) diz respeito à pouca atenção dada ao tipo e grau de DI. Tendo em conta que essa variável é de importância decisiva em termos de dependência, necessidade de atenção, contato com os serviços e preocupações com o futuro (Seligman \& Darling, 2007), deveria ser considerada de uma forma mais consistente pela investigação nessa área.

Têm-se realizado, também, estudos exploratórios com populações mais específicas, (irmãos de pessoas com DI) através de métodos qualitativos, tendo-se concluído que surgem domínios distintos da QdVF quando se consideram diferentes grupos (p. ex., irmãos), o que vai ao encontro da existência de especificidades, quando se consideram diferentes elementos da família (Seligman \& Darling, 2007). Também, ao longo das etapas do ciclo de vida da família, surgem diferenças em termos de QdVF, comprovadas através do estudo com pais de pessoas mais velhas com DI e explicadas pelas tarefas desenvolvimentais específicas da DI, associadas a cada uma das etapas (Carr \& O'Reilly, 2007; Seligman \& Darling, 2007). Tais fatos mostram que recolher informação apenas com o cuidador principal ou um dos pais da pessoa com DI e unicamente num momento pontual poderá ser insuficiente para conhecer as perceções de toda a família acerca da sua qualidade de vida e ao longo do seu ciclo de vida.

Em suma, os estudos acerca da QdVF em famílias de pessoas com DI demonstraram que esse é um conceito abrangente e que apresenta muitas potencialidades, pelo que importa dar continuidade a essa linha de investigação, contribuindo para uma melhor compreensão e intervenções que visem o aumento da QdV de pessoas com DI e suas famílias. 


\section{Estudos Futuros}

No que diz respeito aos próximos passos de investigação nessa área e tendo em conta que o desenvolvimento de instrumentos que medem a QdVF partiu essencialmente de estudos empíricos (Hu, Summers, Turnbull, \& Zuna, 2011), importa consolidar os instrumentos de avaliação existentes no sentido de os melhorar, baseando-os em teorias robustas. Ainda no que diz respeito aos instrumentos existentes, deverá incluir-se a avaliação das dinâmicas familiares de forma a conhecerem-se as interações entre os diferentes elementos da família no que diz respeito à QdVF (Hu et al., 2011), não se limitando a uma avaliação individual que represente a perceção de cada um dos elementos da família. Do ponto de vista metodológico, seria importante recorrer quer a medidas objetivas, quer subjetivas e a designs de investigação multivariados, para avaliar a forma como as características pessoais, as variáveis ambientais e a QdV dos elementos da família se relacionam com a QdVF (Samuel, Rillotta \& Brown, 2012).

Tendo em conta que uma das limitações presentes nos estudos analisados diz respeito ao fato de a maioria dos mesmos apenas se focar na percepção do cuidador principal (que habitualmente é um dos pais da pessoa com DI; Brown et al., 2016), sugerem-se, como estudos futuros, investigações que procurem conhecer as percepções dos diferentes elementos da família.

Finalmente, uma vez que os estudos têm mostrado a presença de diferenças, em termos de QdVF em função da etapa do ciclo de vida da família, importa analisar as especificidades das diferentes etapas, a fim de explorar o modo como as tarefas desenvolvimentais correspondentes a cada etapa influenciam a avaliação da QdVF em famílias de pessoas com DI.

\section{REFERÊNCIAS}

Ajuwon, P., \& Brown, I. (2012). Family quality of life in Nigeria. Journal of Intellectual Disability Research, 56(1), 61-70. doi: 10.1111/j.1365-2788.2011.01487.x

American Association of Intellectual and Developmental Disabilities (2011). Discapacidad intelectual: Definicion, clasificacion y sistemas de apoyo (11 ${ }^{\mathrm{a}} \mathrm{ed} ., \mathrm{M}$. Verdugo, Trad.). Madrid: Alianza.

Aznar, A., \& Castañón, D. (2005). Quality of life from the point of view of Latin American families: A participative research study. Journal of Intellectual Disability Research, 49(10), 784-788. doi: 10.1111/j.1365-2788.2005.00752.x

Balcells-Balcells, A., Giné, C., Guàrdia-Olmos, J., \& Summers, J.A. (2011). Family quality of life: Adaptation to Spanish population of several family support questionnaires. Journal of Intellectual Disability Research, 55(12), 1151-1163. doi:10.1111/j.13652788.2010.01350.x

Baum, S. (2006). The use of systemic approach to adults with intellectual disabilities and their families: Historical overview and current research. In S. Baum \& H. Lynggaard (Eds.), Intellectual disabilities: A systemic approach (pp. 21-41). London: Karnac.

Bertelli, M., Bianco, A., Rossi, M., Scuticchio, D., \& Brown, I. (2011). Relationship between individual quality of life and family quality of life for people with intellectual disability living in Italy. Journal of Intellectual Disability Research, 55(12), 1136-1150. doi: 10.1111/j.1365-2788.2011.01464.x

Bronfenbrenner, U. (1977). Toward an experimental ecology of human development. American Psychologist, 32(7), 513-531.

Brown, I., \& Brown, R. (2004a). Family quality of life as an area of study. In A. Turnbull, I. Brown, \& R. Turnbull (Eds.), Families and persons with mental retardation and quality of life: International perspectives (pp. 3-10). Washington: American Association on Mental Retardation.

Brown, I., \& Brown, R. (2004b). Concepts for beginning study in family quality of life. In A. Turnbull, I. Brown, \& R. Turnbull (Eds.), Families and persons with mental retardation and quality of life: International perspectives (pp. 25-47). Washington: American Association on Mental Retardation.

Brown, I., Anand, S., Fung, W.L., Isaacs, B., \& Baum, N. (2003). Family quality of life: Canadian results from an international study. Journal of Developmental and Physical Disabilities, 15(3), 207-230.
Brown, R., MacAdam-Crisp, J., Wang, M., \& Iarocci, G. (2006). Family quality of life when there is a child with a developmental disability. Journal of Policy and Practice in Intellectual Disabilities, 3(4), 238-245. doi: 10.1111/j.17411130.2006.00085.x

Brown, R., Kyrkou, M., \& Samuel, P. (2016). Family quality of life. In I. Rubin, J. Merrick, D. Greydanus, \& D. Patel (Eds.), Health care for people with intellectual and developmental disabilities across the lifespan (pp. 2065-2082). Cham: Springer International Publishing.

Čagran, B., Schmidt, M., \& Brown, I. (2011). Assessment of the quality of life in families with children who have intellectual and developmental disabilities in Slovenia. Journal of Intellectual Disability Research, 55(12), 1164-1175. doi: 10.1111/j.1365-2788.2011.01400.x

Carr, A., \& O'Reilly, G. (2007). Lifespan development and the family lifecycle. In A. Carr, G. O'Reilly, P. Walsh, \& J. McEvoy (Eds.), The handbook of intellectual disability and clinical psychology practice (pp. 50-91). London: Routledge.

Clark, M., Brown, R., \& Karrapaya, R. (2012). An initial look at the quality of life of Malaysian families that include children with disabilities. Journal of Intellectual Disability Research, 56(1), 45-60. doi: 10.1177/1053815116636885

Davis, K., \& Gavidia-Payne, S. (2009). The impact of child, family, and professional support characteristics on the quality of life in families of young children with disabilities. Journal of Intellectual \& Developmental Disability, 34(2), 153-162. doi: $10.1080 / 13668250902874608$

Fredman, G. (2006). Working systemically with intellectual disability: Why not? In S. Baum \& H. Lynggaard (Eds.), Intellectual disabilities: A systemic approach (pp. 1-20). London: Karnac.

Giné, C., Grácia, M., Vilaseca, R., \& Balcells, A. (2010). Quality of life of the families of people with intellectual disability in Spain. In R. Kober (Ed.), Enhancing quality of life for people with intellectual disabilities: From theory to practice (pp. 349-361). New York: Springer.

Giné, C., Vilaseca, R., Grácia, M., Mora, J., Orcasitas, J., Simon, C., ... Simo-Pinatella, D. (2013). Spanish Family Quality of Life Scales: Under and over 18 years old. Journal of Intellectual \& Developmental Disability, 38(2), 141-148. doi: $10.3109 / 13668250.2013 .774324$ 
Hatton, C. (2012). Intellectual disabilities: Classification, epidemiology and causes. In E. Emerson, C. Hatton, K. Dickson, R. Gone, A. Caine, \& J. Bromley (Eds.), Clinical psychology and people with intellectual disabilities (pp. 3-22). Chicester: Wiley-Blackwell.

Ho, E., James, N., Brown, I., Firkowska-Mankiewicz, A., Zasępa, E., Wołowicz, A., \& Wapiennik, E. (2013). Family quality of life of Polish families with a member with intellectual disability. Journal on Developmental Disabilities, 19(2), 36-41.

Hoffman, L., Marquis, J., Poston, D., Summers, J., \& Turnbull, A. (2006). Assessing family outcomes: Psychometric evaluation of the Beach Center Family Quality of Life Scale. Journal of Marriage and Family, 68, 1069-1083. doi: 10.1111/j.17413737.2006.00314.x

Hu, X., Summers, J., Turnbull, A., \& Zuna, N. (2011). The quantitative measurement of family quality of life: A review of available instruments. Journal of Intellectual Disability Research, 55(12), 1098-1114. doi: 10.1111/j.13652788.2011.01463.x

Hu, X., Wang, M., \& Fei, X. (2012). Family quality of life of Chinese families of children with intellectual disabilities. Journal of Intellectual Disability Research, 56(1), 30-44. doi: 10.1111/j.1365-2788.2011.01391.x

Instituto Nacional para a Reabilitação. (2010). Convenção sobre os Direitos das Pessoas com Deficiência (2 ${ }^{\mathrm{a}} \mathrm{ed}$.). Lisboa: INR.

Isaacs, B. J., Brown, I., Brown, R. I., Baum, N., Myerscough, T., Neikrug, S., ... Wang, M. (2007). The International Family Quality of Life Project: Goals and description of a survey tool. Journal of Policy \& Practice in Intellectual Disabilities, 4(3), 177-185. doi: 10.1111/j.1741-1130.2007.00116.x

Isaacs, B., Wang, M., Samuel, P., Ajuwon, P., Baum, N., Edwards, M., \& Rillotta, F. (2012). Testing the factor structure of the Family Quality of Life Survey - 2006. Journal of Intellectual Disability Research, 56(1), 17-29. doi: 10.1111/j.13652788.2011.01392.x

Jokinen, N. (2006). Family quality of life and older families. Journal of Policy and Practice in Intellectual Disabilities, 3(4), 246252. doi: 10.1111/j.1741-1130.2006.00086.x

Jokinen, N., \& Brown, R. (2005). Family quality of life from the perspective of older parents. Journal of Intellectual Disability Research, 49(10), 789-793. doi: 10.1111/j.13652788.2005.00753.x

Keith, K. (2007). Quality of life. In A. Carr, G. O'Reilly, P. Walsh, \& J.McEvoy (Eds.), The handbook of intellectual disability and clinical psychology practice (pp.143-168). London: Routledge.

Kober, R. (2010). Enhancing the quality of life of people with intellectual disabilities: From theory to practice. New York: Springer.

Leal, L. (2008). Un enfoque de la discapacidad intelectual centrado en la familia (M. Ramón \& E. Fernández, Trad). Madrid: FEAPS.

McFelea, J., \& Raver, S. (2012). Quality of life of families with children who have severe developmental disabilities: A comparison based on child residence. Physical Disabilities: Education and Related Services, 31(2), 3-17.

Moyson, T., \& Roeyers, H. (2012). 'The overall quality of my life as a sibling is all right, but of course, it could always be better'. Quality of life of siblings of children with intellectual disability: The siblings' perspectives. Journal of Intellectual Disability Research, 56(1), 87-101. doi: 10.1111/j.13652788.2011.01393.x

Neikrug, S., Roth, D., \& Judes, J. (2011). Lives of quality in the face of challenge in Israel. Journal of Intellectual Disability Research, 55(12), 1176-1184. doi: 10.1111/j.13652788.2011.01475.x

Park, J. J., Hoffman, L. L., Marquis, J. J., Turnbull, A. P., Poston, D. D., Mannan, H. H., ... Nelson, L. L. (2003). Toward assessing family outcomes of service delivery: Validation of a family quality of life survey. Journal of Intellectual Disability Research, 47(4/5), 367-384.

Poston, D., Turnbull, A., Park, J., Mannan, H., Marquis, J., \& Wang, M. (2003). Family quality of life: A qualitative inquiry. Mental Retardation, 41(5), 313-328.

Rillotta, F., Kirby, N., \& Shearer, J. (2010). A Comparison of two family quality of life measures: An Australian study. In R. Kober (Ed.), Enhancing quality of life for people with intellectual disabilities: From theory to practice (pp. 305-348). New York: Springer.

Rillotta, F., Kirby, N., Shearer, J., \& Nettelbeck, T. (2012). Family quality of life of Australian families with a member with an intellectual/developmental disability. Journal of Intellectual Disability Research, 56(1), 71-86. doi: 10.1111/j.13652788.2011.01462.x

Samuel, P., Hobden, K., LeRoy, B., \& Lacey, K. (2012). Analysing family service needs of typically underserved families in the USA. Journal of Intellectual Disability Research, 56(1), 111128. doi: 10.1111/j.1365-2788.2011.01481.x

Samuel, P., Rillotta, F., \& Brown, I. (2012). The development of family quality of life concepts and measures. Journal of Intellectual Disability Research, 56(1), 1-16. doi: 10.1111/j.1365-2788.2011.01486.x

Schalock, R. (2004). Moving from individual to family quality of life as a research topic. In A. Turnbull, I. Brown, \& R. Turnbull (Eds.), Families and persons with mental retardation and quality of life: International perspectives (pp. 11-24). Washington: American Association on Mental Retardation.

Schalock, R. L., Brown, I., Brown, R., Cummins, R. A., Felce, D., Matikka, L., ... Parmenter, T. (2002). Conceptualization, measurement, and application of quality of life for persons with intellectual disabilities: Report of an international panel of experts. Mental Retardation, 40(6), 457-470.

Schalock, R., \& Verdugo, M. (2003). Calidad de vida: Manual para profesionales de la educación, salud y servicios sociales (M. Verdugo \& C. Río, Trad.). Madrid: Alianza.

Seligman, M., \& Darling, R. (2007). Ordinary families, special children: A systems approach to childhood disability ( $3^{\mathrm{a}}$ ed.). New York: The Guilford Press.

Steel, R., Poppe, L., Vandevelde, S., Van Hove, G., \& Claes, C. (2011). Family quality of life in 25 Belgian families: Quantitative and qualitative exploration of social and professional support domains. Journal of Intellectual Disability Research, 55(12), 1123-1135. doi: 10.1111/j.13652788.2011.01433.x

Summers, J. A., Poston, D. J., Turnbull, A. P., Marquis, J. J., Hoffman, L. L., Mannan, H. H., \& Wang, M. M. (2005). Conceptualizing and measuring family quality of life. Journal of Intellectual Disability Research, 49(10), 777-783.

Sung, M., \& Park, J. (2012). The effects of a family support program including respite care on parenting stress and family quality of life perceived by primary caregivers of children with disabilities in Korea. International Journal of Special Education, 27(3), 188-198.

Svraka, E., Loga, S., \& Brown, I. (2011). Family quality of life: Adult school children with intellectual disabilities in Bosnia and Herzegovina. Journal of Intellectual Disability Research, 55(12), 1115-1122. doi: 10.1111/j.1365-2788.2011.01434.x

Tamarit, J. (2006). Calidad FEAPS: Un modelo para la calidad de vida, desde la ética y la calidad del servicio. In M. Verdugo, \& F. Veja (Coords.), Rompiendo inercias. Claves para avanzar (pp. 163-178). Salamanca: Amarú Ediciones.

Verdugo, M., Córdoba, L., \& Gómez, J. (2005). Spanish adaptation of the Family Quality of Life Survey. Journal of Intellectual Disability Research, 49(10), 794-798.

Verdugo, M., Schalock, R., Keith, K., \& Stancliffe, R. (2005). Quality of life and its measurement: Important principles 
and guidelines. Journal of Intellectual Disability Research, 49(10), 707-717.

Wang, M., Summers, J., Little, T., Turnbull, A., Poston, D., \& Mannan, H. (2006). Perspectives of fathers and mothers of children in early intervention programmes in assessing family quality of life. Journal of Intellectual Disability Research, 50(12), 977-988.

Wang, M., Turnbull, A., Summers, J., Little, T., Poston, D., Mannan, H., \& Turnbull, R. (2004). Severity of disability and income as predictors of parents' satisfaction with their family quality of life during early childhood years. Research \& Practice for Persons with Severe Disabilities, 29(2), 82-94. doi: 10.2511/ rpsd.29.2.82

Werner, S., Edwards, M., \& Baum, N. (2009). Family quality of life before and after out-of-home placement of a family member with an intellectual disability. Journal of Policy and Practice in Intellectual Disabilities, 6(1), 32-39. doi: 10.1111/j.17411130.2008.00196.x

Werner, S., Edwards, M., Baum, N., Brown, I., Brown, R., \& Isaacs, B. (2009). Family quality of life among families with a member who has an intellectual disability: An exploratory examination of key domains and dimensions of the revised FQOL Survey. Journal of Intellectual Disability Research, 53(6), 501-511. doi: 10.1111/j.1365-2788.2009.01164.x

Zuna, N. I., Summers, J. A., Turnbull, A. P., Hu, X., \& Xu, S. (2010). Theorizing about family quality of life. In R. Kober (Ed.), Enhancing quality of life for people with intellectual disabilities: From theory to practice (pp. 241-278). New York: Springer.

Zuna, N. I., Turnbull, A. A., \& Summers, J. A. (2009). Family quality of life: Moving from measurement to application. Journal of Policy and Practice in Intellectual Disabilities, 6(1), 25-31. doi: 10.1111/j.1741-1130.2008.00199.x 